\title{
FUNGOS ASSOCIADOS A INVERTEBRADOS MARINHOS: ISOLAMENTO, SELEÇÃO E AVALIAÇÃO DA PRODUÇÃO DE ENZIMAS CELULOLÍTICAS
}

Dissertação apresentada ao Programa de Pós-Graduação Interunidade em Biotecnologia USP/Instituto Butantan/ IPT, para a obtenção do Título de Mestre em Biotecnologia.

Área de Concentração: Biotecnologia

Orientadora: Prof.(a). Dr(a). Lara Durães Sette

Co-Orientadora: Prof.(a). $\operatorname{Dr}(\mathrm{a})$. Maria Filomena de Andrade Rodrigues 


\section{RESUMO}

SILVA, C. H. D. Fungos associados a invertebrados marinhos: isolamento, seleção e avaliação da produção de enzimas celulolíticas, 2010. $69 \mathrm{f}$. Dissertação (Mestrado em Biotecnologia) - Instituto de Ciências Biomédicas, Universidade de São Paulo, São Paulo, 2010.

Os fungos filamentosos derivados de ambiente marinho têm sido foco de grande interesse devido principalmente ao fato da micologia marinha ser uma ciência relativamente recente e de pouco se conhecer sobre a diversidade filogenética e funcional das comunidades microbianas marinhas. Portanto, 0 isolamento, triagem e preservação de fungos derivados do mar podem levar à descoberta de novas moléculas ativas e/ou enzimas extracelulares de aplicação em diversos setores de importância econômica. Entretanto, os trabalhos de pesquisa envolvendo a microbiologia marinha são ainda escassos. Neste contexto, o objetivo deste estudo foi conhecer a diversidade de fungos filamentosos derivados marinhos, bem como, selecionar isolados capazes de produzir enzimas celulolíticas (CMCase, FPase e Celobiase). Para tanto, foram utilizados 86 fungos filamentosos isolados seletivamente (meio AC) a partir de amostras de macro-organismos marinhos coletadas no litoral norte do estado de São Paulo em janeiro de 2007 (primeira coleta) e dezembro de 2008 (segunda coleta). Os resultados demonstraram a presença de uma ampla diversidade de fungos potencialmente celulolíticos, visto que dos 30 isolados derivados da primeira coleta, foram obtidos 25 ripotipos distintos. Em adição, dados derivados das análises taxonômicas realizadas para 38 isolados revelaram a presença de um fungo pertencente ao filo Basidiomycota e 37 ao filo Ascomycota (distribuídos em oito diferentes ordens e 17 gêneros). Dentre os 86 fungos submetidos aos experimentos de produção de celulases, 17 apresentaram resultados satisfatórios de CMCase e FPase (em meio líquido após 14 dias de cultivo) e foram selecionados para a avaliação da atividade de Celobiase. Os isolados F188 (Didymella sp.) e CiAC 7 (Aspergillus sp.) apresentaram os melhores resultados de produção de celulases e foram submetidos ao experimento de cinética enzimática (durante 7 dias), utilizando fontes de carbono natural (farelo de trigo) e sintética (Avicel). De maneira geral, os melhores resultados de produção de celulases foram obtidos em meio contendo farelo de trigo e, nestas condições, o isolado CiAC 7 (Aspergillus sp.) apresentou a maior atividade de Celobiase (0,055 U/mL.min). Os resultados do presente trabalho demonstram o potencial para aplicação biotecnológica de fungos derivados de ambiente marinho e estimulam o desenvolvimento de novos estudos de produção e caracterização de celulases. Em adição, os isolados que apresentaram potencial para produção de celulases foram depositados na Coleção Brasileira de Micro-organismos de Ambiente e Indústria (CBMAI).

Palavra-chave: Fungos marinhos. Macro-organismos marinhos. Enzimas celulolíticas. 


\section{ABSTRACT}

SILVA, C. H. D. Fungi associated with marine invertebrates: isolation, selection and evaluation of production of cellulytic enzymes. 2010. 69 p. Dissertation (Biotecnology) - Instituto de Ciências Biomédicas, Universidade de São Paulo, São Paulo, 2010.

The filamentous fungi derived from marine environment have been focus of great interest, mainly due to the fact that marine mycology is a relatively new science and that little is known about the phylogenetic and functional diversity of marine microbial communities. Therefore, the isolation, screening and preservation of marine-derived fungi can lead to the discovery of new active molecules and/or extracellular enzymes for being applied in several economic sectors. However, researches involving marine microbiology are still scarce. In this context, the aim of this study was to characterize the marine-derived filamentous fungi diversity, as well as, to screen fungal isolates able to produce cellulolytic enzymes (CMCase, FPase and Celobiase). For that end, were used 86 filamentous fungi selectively isolated (AC medium) from samples of marine macro-organisms collected on the northern coast of São Paulo State in January 2007 (first sampling) and December 2008 (second sampling). The results showed the presence of a great diversity of potential cellulolytic filamentous fungi, since among the 30 isolates recovered from the first sampling, 25 distinct ripotypes were obtained. In addition, data derived from the taxonomic analyses, performed for 38 isolates, revealed the presence of one fungus affiliated to the phylum Basidiomycota and 37 to the phylum Ascomycota (distributed among eight different orders and 17 genera). Among the 86 fungal isolates that were submitted to the experiments of cellulases production, 17 presented satisfactory results of CMCase and FPase (in liquid medium after 14 days of cultivation) and were selected for cellobiase activity evaluation. Fungal isolates F188 (Didymella sp.) and CIAC7 (Aspergillus sp.) presented the better results of cellulases production and were submitted to the enzyme kinetics experiment (during seven days), using natural (wheat bran) and synthetic (Avicel) carbon sources. In general, better results of cellulases production were obtained in medium containing wheat bran and, under these conditions, the fungal isolate CIAC 7 (Aspergillus sp.) showed the highest activity of cellobiase (0.055 U / mL.min). Results from the present work demonstrate the potential of fungi derived from marine environment for biotechnological application and encourages the development of further studies related to cellulases production and characterization. In addition, strains that showed potential for cellulases production were deposited in the Brazilian Collection of Environmental and Industrial Microorganisms (CBMAI)

Keyword: Marine fungi. Marine macroorganisms. Cellulolytic enzymes. 


\section{INTRODUÇÃO}

Os fungos filamentosos derivados de ambiente marinho têm sido foco de grande interesse devido principalmente ao fato da micologia marinha ser uma ciência relativamente recente e de pouco se conhecer sobre a diversidade filogenética e funcional das comunidades microbianas marinhas. Portanto, a exploração da biodiversidade marinha como fonte de potenciais agentes quimioterapêuticos, agroquímicos ou para obtenção de produtos biotecnológicos, é extremamente promissora. Os metabólitos de origem marinha ganharam significativa importância durante as duas últimas décadas, pelo fato de apresentarem atividades biológicas diversificadas bem como características estruturais únicas. Atualmente, as enzimas e os metabólitos produzidos por fungos filamentosos derivados de ambientes marinhos têm sido foco de grande interesse, visto que o oceano cobre mais de $70 \%$ da superfície do planeta e por representar um ambiente pouco explorado com relação à sua biodiversidade e recursos genéticos. Estima-se que a diversidade biológica dos ecossistemas marinhos é maior do que em florestas tropicais (LARSEN et al., 2005).

No ambiente marinho os fungos filamentosos possuem importante papel como decompositores de matéria orgânica vegetal (BUGNI e IRELAND, 2004). A biodegradação de matérias lignocelulolíticas constitui num dos mais importantes ciclos de carbono na natureza, e tendo em vista a importância econômica das celulases, principalmente para os setores têxtil, alimentício, papeleiro e energético (combustível), somando ao fato de que os fungos têm se mostrado de extrema importância em processos biotecnológicos, 0 isolamento e seleção de fungos associados à ambiente marinho, visando avaliação do potencial de produção de enzimas celulolíticas pode ser considerado estrategicamente interessante.

E levando-se em consideração o fato de que a conservação e a utilização sustentável da diversidade biológica requer um conhecimento abrangente sobre a riqueza de espécies, abundância e distribuição, e que pouco se sabe sobre a diversidade biológica e funcional das comunidades marinhas microbiana. Estudos envolvendo o isolamento, triagem e preservação de fungos derivados do mar podem levar à descoberta de novas moléculas ativas e/ou enzimas extracelulares de aplicação em diversos setores de importância sócio-econômica.

Neste contexto, o presente trabalho teve como objetivo principal a obtenção de isolados de fungos filamentosos, derivados de ambiente marinho, produtores de 
celulases. Para tanto, foi realizado um isolamento seletivo dos fungos associados a diferentes amostras de macro-organismos marinhos (algas, esponjas, estrela-do-mar e ascídia), avaliação da produção de enzimas celulolíticas, identificação taxonômica dos isolados selecionados e depósito na Coloeção de Micro-organismos Marinhos estruturada no âmbito da Coleção Brasileira de micro-organismos de Ambiente e Indústria (CBMAI) do CPQBA/UNICAMP. 


\section{CONCLUSÃO}

O uso da técnica de isolamento seletivo para obtenção de fungos marinhos produtores de celulases foi aplicada com sucesso no presente estudo, visto que dos 35 isolados avaliados quanto à produção de CMCase em meio sólido, 77\% apresentou halo de degradação. Foram isolados no total 81 fungos filamentosos (13 a partir de amostras de macro-organismos marinhos e um da água do mar).

A utilização da técnica de ARDRA juntamente com a caracterização taxonômica molecular revelou uma ampla diversidade de fungos filamentosos potencialmente celulolíticos associados a algas e invertebrados marinhos. Do total de 38 fungos utilizados nos estudos de diversidade um isolado foi afiliado ao filo Basidiomycota, sendo os 37 restantes representantes do filo Ascomycota, incluindo 8 diferentes ordens e 17 gêneros distintos. Os invertebrados marinhos Didemnum sp., Axinella corrugata, Amphimedon viridis e Dragmacidon reticulata foram os que derivaram o maior número de isolados.

Dentre os 83 fungos derivados-marinhos submetidos aos experimentos de avaliação das atividades enzimáticas de CMCase e FPase, a maioria apresentou resultado positivo de produção para ambas as enzimas, porém nenhum deles foi capaz de produzir quantidades superiores às produzidas pelo fungo Thrichoderma reesei RUT C30, utilizado como padrão. Por outro lado, seis isolados apresentaram atividade superior ao do fungo padrão para a enzima Celobiase: F188 (Didymella sp.), F159 (Fusarium sp.), F75 (Aspergillus sp.), F187 (Fusarium sp.), CiAC 7 (Aspergillus sp.) e $\operatorname{Dr}(6)$ AC 43 (Trichoderma sp.), com destaque para o isolado F188, que apresentou atividade 4,5 vezes maior do que a linhagem padrão.

Os resultados derivados dos experimentos de cinética enzimática para os fungos F188 (Didymella sp.) e CiAC 7 (Aspergillus sp.) revelaram o que a atividade celulolítica foi influenciada pela fonte de carbono utilizada. O farelo de trigo (fonte natural de carbono) derivou os melhores resultados de produção enzimática, sendo que nestas condições o fungo CiAC 7 (Aspergillus sp.) apresentou o melhor resultado de produção de Celobiase (0,055 U/mL.min) após 5 dias de cultivo.

Os isolados F188 (Didymella sp.) e CiAC 7 (Aspergillus sp.), bem como outros fungos estudados no presente trabalho, podem ser considerados como potenciais fontes de recursos genéticos microbianos a ser biotecnologicamente explorados e utilizados pelos setores têxtil, alimentício, papeleiro e de biocombustíveis. 
De maneira geral, podemos concluir que os objetivos propostos pelo presente trabalho foram concluídos com êxito. A combinação dos dados obtidos contribui para ampliação do conhecimento da diversidade de fungos filamentosos associada ao ecossistema marinho e do potencial biotecnológico destes isolados, principalmente na degradação da celulose. Em adição, o depósito dos isolados produtores de celulases na Coleção Brasileira de Micro-organismos de Ambiente e Indústria (CBMAI), permitirá que estudos envolvendo outras aplicações biotecnológicas possam ser realizados. 


\section{REFERÊNCIAS ${ }^{1}$}

ABARCA, M. L. et al. Taxonomy and sigificance of black aspergilli. Antonie van Leeuwenhoek, v. 86, p. 33-49, 2004.

BAKER, P. W. et al. Phylogenetic diversity and antimicrobial activities of fungi associated with Haliclona simulans isolated from Irish coastal waters. Marine Biotechnology,v. 11, 2008.

BERLINCK, R. G. S. et al. Challenges and Rewards of Research in Marine Natural Products Chemistry in Brazil. Journal of Natural Products. v. 67. n. 3, 2004.

BHAT, M. K. Cellulases and related enzymes in biotechnology. Biotechnology Advances [S.I.], v. 18, p. 355-383, 2000.

BLUNT, J. W. et al. Marine Natural Produtcts. Natural Product Reports, v. 22, p. 15-61, 2005 .

BONUGLI-SANTOS, R. C. et al. Production of laccase, manganese peroxidase and lignin peroxidase by Brazilian marine-derived fungi. Enzyme Microbial Technology, v. 46, 2009.

BRIDGE, P.; SPOONER, B. Soil fungi: diversity and detection. Plant and Soil. v. 232, p. 147- 154, 2001.

BUGNI, T. S.; IRELAND, C. M. Marine-derived fungi: a chemically and biologically diverse group of microorganisms. Natural Product Reports , p. 143-163, 2004.

BÉGUIM, P.; AUBERT, J. P. Cellulases. In: LEDERBERG, J. (Ed.). Encyclopedia of Microbiology. v. 1. New York, N. Y.: Academic Press, p. 467 -477, 1992.

COUGHLAN, M. P. The properties of Fungal and Bacterial Cellulases with Comment on their Production and Application. In: RUSSEL, E. G. (Ed.). Biotechnology \& Genetic Engeneering Reviews. v. 3. New Castle: Intercept Portland Limited, p. 39-109, 1982.

DA SILVA, M. et al. Cnidarian-derived filamentous fungi from Brazil: isolation, characterization and RBBR decolourization screening. Environ Technology, p. 1331-9, 2008.

DEKKER, R. R. H. Biodegradation of the Hemicellulose. Biosynthesis and Biodegradation of wood Components. v. 18: Academic Press Inc., p. 505-533, 1985.

ESPOSITO, E.; AZEVEDO, J. L. D. Fungos: uma intrudução à biologia, bioquimica e biotecnologia. Caxias do Sul: Educs, 2004.

FAN, L. T. et al. Cellulose Hydrolysis. New York: Springer-Verlag, 1987.

FENICAL, W.; JENSEN, P. R. Strategies for the discovery of secondary metabolites from marine bacteria: ecological perspective. Annual Reviews in Microbiology, v. 48, p. 559-584, 1994.

\footnotetext{
${ }^{1}$ De acordo com:

International Comittee of Medical Journal Editors. Uniform requirements for manuscripts submitted to Biomedical Journal: sample references Available from: http://www.icmje.org [2007 may 22]
} 
GAO, Z. et al. Molecular Detection of Fungal Communities in the Hawaiian Marine Sponges Suberites zeteki and Mycale armata. Applied and Environmental Microbiology.,v. 74, p. 6091 $-6101,2008$.

GHOSE, T. K. et al. MEASUREMENT OF CELLULASE ACTIVITIES. Pure \& Applied. Chemistry. v. 59. n. 2. Great Britain, p. 257-268, 1987.

GHOSH, A. et al. Cellulase secretion from a hyper-cellulolytic mutant of Trichoderma reesei Rut-C30. Archives of Microbiology. v. 140. n. 2, p. 126-133, 1984.

GOLUBIC, S. et al. Endolithic fungi in marine ecosystems. Trends in Microbiology. v. 13. n. $5,2005$.

HAWKSWORTH, D. L.; ROSSMAN, A. Y. Where Are All the Undescribed Fungi? Phytopathology, p. 888-891, 1997.

HILL, R. A. Marine natural products. Annual. Reports. Program. Chemistry. v. 101, p. 124$136,2005$.

KIMURA, M. A simple method for estimating evolutionary rates of base substitutions through comparative studies of nucleotide sequences. Journal of Molecular Evolution, p. 111$120,1980$.

KNAPP, J. S. Biodegradation of Cellulose and Lignin. Comprehensive Biotechnology. v. 3. New York: Pergamon Press Inc., p. 835-846, 1985.

KOHLMEYER, J.; KOHLMEYER, E. Marine Micology: The Higher Fungi. Academic Press. New York, 1979.

KUBICEK, C. P. The Cellulose Proteins of Trichoderma reesei: Structures Multiplicity, Mode of Action and Regulation of Formatation. Biochemistry Engeneering/ Biotechnology. v. 45, p. 1-27, 1992.

KURTZMAN, C. P.; ROBNETT, C. J. Identification and phylogeny of ascomycetous yeasts from analysis of nuclear large subunit (26S) ribosomal DNA partial sequences. Antonie van Leeuwenhoek, p. 331-371, 1998.

LARSEN, T. O. et al. Phenotypic taxonomy and metabolite profiling in microbial drug discovery. Natural Products Report. v. 22, p. 672-95, 2005.

LE CROM, S. et al. Tracking the roots of cellulase hyperproduction by the fungus Trichoderma reesei using massively parallel DNA sequencing. Proceeding of the National Academy of Sciences, p. 16151-6, 2009.

LIBERRA, K.; LINDERQUIST, U. Marine fungi-a prolific resource of biologically active natural products? Pharmazie. v. 50, p. 583-588, 1995.

MANDELS, M. Application of cellulases. Biochemical society transactions. v. 13, p. 414416, 1985.

MENDES, E. V. A atenção primária à saúde no SUS. Ceará: Escola de Saúde Pública, 2002. 
MENEZES, C. B. A. et al. Microbial diversity associated with algae, ascidians and sponges from the north coast of São Paulo state, Brazil. Microbiological Research. v. In Press 2009.

MONSALVE, G. et al. Producción de etanol a partir de la cáscara de banano y de almidón de yuca. Dyna revista de la.facultad.nacional de.minas. v. 73. n. 150. Medellín, p. 21-27, 2006.

OSTERHAGE, C. Isolation, Structure Determination and Biological Activity Assessment of Secondary Metabolites from Marine-derived Fungi. Alemanha, 2001.

PAVARINA, E. C. Estudo dos sistemas celulolíticos e fermentativos de fungos microaróbios facultativos. Tese de Mestrado- Faculdade de Engenharia de Alimentos, Unicamp, Campinas, 1997.

PEI-CHIH, W. et al. Characteristics of indoor and outdoor airbome fungi at suburban and urban homes in two seasons. Science of the Total Environment. v. 253, p. 111-118, 2000.

PRASANNARAI, K.; SRIDHAR, K. R. Diversity and abundance of higher marine fungi on woody substrates along the west coast of India. CURRENT SCIENCE . v. 88. n. 3, 2001. p. 30411 .

QIANQUN, G. et al. Recent Researches of Bioactive Metabolites in Marine Organismsassociated Microorganisms. Journal of Ocean University of China. v. 3. n. 2, p. 150-156, 2004.

RAEDER, U.; BRODA, P. Rapid preparation of DNA from filamentous fungi. Letters in Applied Microbiology, v. 1, p. 17-20, 1985.

ROMERO, F. et al. Thiocoraline, a new despsipeptide with antitumor activity produced by a marine Micromonospora. Jornal. Antibiotics. v. 50, p. 734-737, 1997.

RUEGGER, M. J. S.; TAUK-TORNISIELO, S. M. Cellulase activity of fungi isolated from soil of the Ecological Station of Juréia-Itatins, São Paulo, Brazil. Revista. Brasileira de Botanica. v. 27. n. 2. São paulo, 2004.

SERMANNI, G. G.; PORRI, A. The potentiality of solid state biotransformation of lignocellulosic materiais. Chimica Oggi, p. 15 - 19, 1989.

SETTE, L. D. et al. Isolation and characterization of alachlor-degrading actinomycetes from soil. Antonie van Leeuwenhoek. v. 87, p. 81-89, 2005.

SURAJIT, D. et al. Marine microbial diversity and ecology: importance and future perspectives. Current Science. v. 90. n. 10, 2006.

SUTAR, R. et al. Comparasion of ITS and IGS regions for strain typing of clinical and nonclinical isolates of Pichia anomal. Journal of Medical Microbiology. v. 53, p. 1-5, 2004.

TAMURA, K. et al. MEGA4: Molecular Evolutionary Genetics Analysis (MEGA) software version 4.0. Molecular biology and evolution, p. 1596-9, 2007.

THOMPSON, J. D. et al. CLUSTAL W: improving the sensitivity of progressive multiple sequence alignment through sequence weighting, position-specific gap penalties and weight matrix choice. Nucleic acids research, p. 4673-80, 1994. 
TORTORA, G. J. et al. Microbiologia. Artmed. Porto Alegre, p. 827, 2000.

VOIGT, K. et al. Phylogeny and PCR Identification of Clinically Important Zygomycetes Based on Nuclear Ribosomal-DNA Sequence Data. Journal of Clinical Microbiology. v. 37. n. 12, p. 3957-3964, 1999.

WANG, G. Diversity and biotechnological potential of the sponge-associated microbial consortia. Journal of Industrial Microbiology and Biotechnology. v. 33, p. 545-51, 2006.

WATSON, T. G. et al. Cellulase production by Trichoderma reesei (RUT-C30) in fed-batch culture. Biotechnology Letters. v. 6. n. 10, p. 667-672, 1984.

WOOD, T. M.; MCCRAE, S. I. Synergism Between Enzymes Involved in the Solubilization of Native Cellulose. Advances in Chemistry. v. 181, p. 181-209, 1979. 